\title{
Exercise tolerance can explain the obesity paradox in patients with systolic heart failure: data from the MECKI Score Research Group
}

\author{
Massimo F. Piepoli1 ${ }^{1}$, Ugo Corrà ${ }^{2}$, Fabrizio Veglia ${ }^{3}$, Alice Bonomi ${ }^{3}$, \\ Elisabetta Salvioni ${ }^{3}$, Gaia Cattadori ${ }^{3,4}$, Marco Metra ${ }^{5}$, Carlo Lombardi ${ }^{5}$, \\ Gianfranco Sinagra ${ }^{6}$, Giuseppe Limongelli ${ }^{7}$, Rosa Raimondo ${ }^{8}$, Federica Re ${ }^{9}$, \\ Damiano Magrì ${ }^{10}$, Romualdo Belardinelli ${ }^{11}$, Gianfranco Parati ${ }^{12}$, Chiara Minà ${ }^{13}$, \\ Angela B. Scardovi ${ }^{14}$, Marco Guazzi ${ }^{15}$, Mariantonietta Cicoira ${ }^{16}$, \\ Domenico Scrutinio ${ }^{17}$, Andrea Di Lenarda ${ }^{18}$, Maurizio Bussotti ${ }^{19}$, Maria Frigerio ${ }^{20}$, \\ Michele Correale ${ }^{21}$, Giovanni Quinto Villani ${ }^{1}$, Stefania Paolillo22, \\ Claudio Passino 3,23,24, Piergiuseppe Agostoni, ${ }^{3,25 *}$, and on behalf of the MECKI \\ Score Research Group ${ }^{\dagger}$
}

\begin{abstract}
${ }^{1}$ Heart Failure Unit, Cardiology Department, G da Saliceto Hospital, Piacenza, Italy; ${ }^{2}$ Divisione di Cardiologia Riabilitativa, Fondazione Salvatore Maugeri, IRCCS, Istituto Scientifico di Veruno, Veruno, Italy; ${ }^{3}$ Centro Cardiologico Monzino, IRCCS, Milano, Italy; ${ }^{4}$ Unità Operativa Cardiologia Riabilitativa, Ospedale S.Giuseppe, Multimedica Spa, IRCCS, Milano, Italy; ${ }^{5}$ Cardiology, Department of Medical and Surgical Specialties, Radiological Sciences, and Public Health, University of Brescia, Italy; ${ }^{6}$ Cardiovascular Department, Ospedali Riuniti and University of Trieste, Trieste, Italy; ${ }^{7}$ Cardiologia SUN, Ospedale Monaldi (Azienda dei Colli), Seconda Università di Napoli, Napoli, Italy; ${ }^{8}$ Salvatore Maugeri Foundation, IRCCS, Istituto Scientifico di Tradate, Dipartimento di Medicina e Riabilitazione Cardiorespiratoria Unità Operativa di Cardiologia Riabilitativa, Tradate, Italy; ${ }^{9}$ Cardiology Division, Cardiac Arrhythmia Center and Cardiomyopathies Unit, St.Camillo-Forlanini Hospital, Roma, Italy; ${ }^{10}$ Department of Clinical and Molecular Medicine, La Sapienza University, Roma, Italy; ${ }^{11}$ Cardiologia Riabilitativa, Azienda Ospedali Riuniti, Ancona, Italy; ${ }^{12}$ Department of Health Science, University of Milano Bicocca and Department of Cardiology, S.Luca Hospital, Istituto Auxologico Italiano, Milano, Italy; ${ }^{13}$ ISMETT (Mediterranean Institute for Transplantation and Advanced Specialized Therapies), Palermo, Italy; ${ }^{14}$ UOC Cardiologia Ospedale S. Spirito, Roma, Italy; ${ }^{15}$ Department of Medical Sciences, Cardiology, IRCCS San Donato Hospital, University of Milan, San Donato Milanese, Italy; ${ }^{16}$ Section of Cardiology, Department of Medicine, University of Verona, Italy; ${ }^{17}$ Division of Cardiology, Salvatore Maugeri Foundation, IRCCS, Institute of Cassano Murge, Bari, Italy; ${ }^{18}$ Cardiovascular Center, Health Authority no. 1, Trieste, Italy; ${ }^{19}$ Division of Cardiology, Salvatore Maugeri Foundation, IRCCS, Institute of Milan, Milan, Italy; ${ }^{20}$ Cardiologic Department 'A. De Gasperis', Ospedale Cà Granda-A.O. Niguarda, Milano, Italy; ${ }^{21}$ Department of Cardiology, University of Foggia, Foggia, Italy; ${ }^{22}$ IRCCS SDN Istituto di Ricerca, Napoli, Italy; ${ }^{23}$ Gabriele Monasterio Foundation, CNR-Regione Toscana, Pisa, Italy; ${ }^{24}$ Scuola Superiore S. Anna, Pisa, Italy; and ${ }^{25}$ Department of Clinical Sciences and Community Health, Cardiovascular Section, University of Milano, Milano, Italy
\end{abstract}

Received 29 July 2015; revised 8 January 2016; accepted 9 January 2016

Aims

Obesity has been found to be protective in heart failure (HF), a finding leading to the concept of an obesity paradox. We hypothesized that a preserved cardiorespiratory fitness in obese HF patients may affect the relationship between survival and body mass index (BMI) and explain the obesity paradox in HF.

Methods and results

A total of 4623 systolic HF patients (LVEF $31.5 \pm 9.5 \%$, BMI $26.2 \pm 3.6 \mathrm{~kg} / \mathrm{m}^{2}$ ) were recruited and prospectively followed in 24 Italian HF centres belonging to the MECKI Score Research Group. Besides full clinical examination, patients underwent maximal cardiopulmonary exercise test at study enrolment. Median follow-up was 1113 (553-1803) days. The study population was divided according to $\mathrm{BMI}\left(<25,25-30,>30\right.$ to $\left.\leq 35 \mathrm{~kg} / \mathrm{m}^{2}\right)$ and predicted peak oxygen consumption (peak $\mathrm{VO}_{2},<50 \%, 50-80 \%,>80 \%$ ). Study endpoints were all-cause and cardiovascular deaths including urgent cardiac transplant. All-cause and cardiovascular deaths occurred in $951(28.6 \%, 57.4$ per person-years) and 802 cases (17.4\%, 48.4 per 1000 person-years), respectively. In the high BMI groups, several

*Corresponding author: Centro Cardiologico Monzino, IRCCS, Department of Clinical Sciences and Community Health, Cardiovascular Section, University of Milan, Via Parea, 4, 20138 Milan, Italy. Tel: + 3902 58002772, Fax: +39 02 502008, Email: piergiuseppe.agostoni@unimi.it or piergiuseppe.agostoni@ccfm.it.

${ }^{\dagger}$ The list of members is given in Appendix 1 
prognostic parameters presented better values [LVEF, peak $\mathrm{VO}_{2}$, ventilation/carbon dioxide slope, renal function, and haemoglobin $(P<0.01)$ ] compared with the lower $\mathrm{BMI}$ groups. Both $\mathrm{BMI}$ and peak $\mathrm{VO}_{2}$ were significant positive predictors of longer survival: both higher $\mathrm{BMI}$ and peak $\mathrm{VO}_{2}$ groups showed lower mortality $(P<0.001)$. At multivariable analysis and using a matching procedure (age, gender, LVEF, and peak $\mathrm{VO}_{2}$ ), the protective role of $\mathrm{BMI}$ disappeared.

Conclusion Exercise tolerance affects the relationship between BMI and survival. Cardiorespiratory fitness mitigates the obesity paradox observed in HF patients.

Keywords Exercise tolerance $\bullet$ Cardiopulmonary exercise testing • Heart failure $\bullet$ Prognosis $\bullet$ Matching analysis - MECKI score

\section{Introduction}

Obesity, commonly defined by increased body mass index (BMI), has been found to be protective in heart failure (HF), a finding leading to the accepted concept of an obesity paradox. ${ }^{1-3}$ This somewhat surprising evidence has been analysed and studied under different perspectives in a broad set of HF patients, and it remains substantially unexplained, with competing and complementary views being proposed..$^{1-3}$ It has been suggested that increased levels of serum lipoproteins play a role by counteracting bacterial cytokines and endotoxins, ${ }^{4}$ while low levels of adiponectin ${ }^{5}$ and a decreased response to sympathetic activation ${ }^{6}$ have been associated with a protective background. Independently of the protective neuro-hormonal and inflammatory role that an increased BMI may bear, the multiple interactions between obesity and major clinical determinants of the natural history of the disease are still under scrutiny.

Some investigators have suggested that the obesity paradox may be partly explained by confounding factors. ${ }^{7,8}$ Cardiorespiratory fitness is strongly related to prognosis in healthy individuals and in cohorts with cardiovascular (CV) diseases. ${ }^{9}$ A number of studies have reported the importance of exercise capacity and other cardiopulmonary exercise test (CPET) variables in predicting prognosis in HF. Indeed, the classic cut-off point for peak oxygen consumption (peak $\mathrm{VO}_{2}$ ) of $14 \mathrm{~mL} \mathrm{O} / \mathrm{kg} / \mathrm{min}$ proposed by Mancini et al. ${ }^{10}$ is still frequently used to classify patients with HF into low-risk and high-risk groups. A strong obesity paradox has also been found in cohorts of patients with coronary heart disease, ${ }^{11}$ but it was not present in those with high levels of exercise tolerance. ${ }^{12}$ Similar findings have been more recently reported in systolic HF patients. ${ }^{13}$

We aimed at further investigating the impact of exercise tolerance and cardiorespiratory capacity on the obesity paradox in a larger cohort of systolic HF patients, using a multicentre database based on CPET. ${ }^{13}$

\section{Methods}

\section{Population and study procedures}

We performed a cohort study on 4843 patients with a history of HF with reduced LVEF, enrolled and prospectively followed in 24 Italian HF centres. All patients were derived from the MECKI score database, a database of chronic HF patients that is continuously updated and that allowed us to validate a new prognostic HF risk model, the Metabolic Exercise test data combined with Cardiac and Kidney Indexes (MECKI) score. ${ }^{14}$ For the present analysis, 220 patients with severe pathological obesity, as arbitrarily defined by BMI $>35$, were excluded, reducing the study cohort to 4623 cases.

At enrolment, inclusion and exclusion criteria were evaluated as previously reported, ${ }^{14}$ and clinical history was recorded. Then, physical examination, laboratory analyses, ECG, and transthoracic echocardiography were performed, as previously described. ${ }^{14}$ CPET was performed, depending on the enrolling centre's equipment, using a ramp protocol on an electronically braked cycle-ergometer or a Bruce modified protocol on a treadmill. Peak $\mathrm{VO}_{2}$ data measured at treadmill exercise were reduced by $10 \%$ to allow an appropriate comparison between the two different procedures; the CPET protocol was set to reach peak exercise in $8-12 \mathrm{~min}$, but tests were stopped as patients reported maximal effort, and exercise parameters were calculated as previously described. ${ }^{14}$ Peak $\mathrm{VO}_{2} \%$ of predicted was calculated according to Hansen et al. ${ }^{15}$

\section{Follow-up and study endpoints}

Follow-up was carried out according to the local HF programme, and it ended with the last clinical evaluation or with the patients' death or heart transplantation. If a patient died outside the hospital where they were being followed up, medical records of the event and a report of the cause of death were considered. The primary study endpoint was all-cause death (total mortality) and CV deaths. Urgent cardiac transplant was considered as death both for total and CV mortality. Procedures of data management were performed as previously described. ${ }^{14}$

\section{Data analysis}

Data analysis was performed in two steps. The first part of the analysis considered the entire population ( 4623 patients), which was divided into four groups according to BMI: $<25,25-30,>30$ to $\leq 35$, and $>35 \mathrm{~kg} / \mathrm{m}^{2}$. Subsequently, to assess the role of cardiopulmonary capacity on survival, the study population was also divided into three subgroups according to predicted peak $\mathrm{VO}_{2}(<50,50-80$, and $>80 \%)$. Demographic, laboratory, echocardiographic, CPET, and follow-up data were compared between groups, and Cox univariable and multivariable regression analysis and Kaplan-Meier survival analysis were performed for the previously described study endpoints (CV mortality and total mortality). Kaplan-Meier analysis was arbitrarily truncated at 3 years. 
In the second step, as a confirmation of the first analysis, we performed a 1:1:1 statistical matching between the three BMI classes. A total of 628 patients per group matched for the following arbitrarily selected variables: age \pm 5 years, gender, $L V E F \pm 5$, and peak $\mathrm{VO}_{2} \pm 150$ $(\mathrm{mL} / \mathrm{min})$. Kaplan-Meier survival analysis was then repeated as previously described.

A further analysis of the BMI $<25$ group, differentiating $\mathrm{BMI}<18.5$ and 18.5-25 groups, was also performed; however, since the percentage of patients with $\mathrm{BMI}<18.5$ was very limited $(<2 \%)$, and thus not comparable with the other groups, no further statistical evaluation was performed (the analysis is presented as Supplementary material online, Tables and Figure S1).

\section{Statistical analysis}

Numerical variables were summarized as mean \pm standard deviation. Analysis of variance (ANOVA) was used when appropriate for between-group comparison. Skewed data are reported as median and interquartile range and compared by Kruskall-Wallis test. Categorical variables, expressed as percentage or frequency, were compared by $\chi^{2}$ test. Bonferroni correction was employed to account for multiple comparisons.

Two multivariable Cox proportional hazard models were used for assessing the independent prognostic value of BMI: the first one adjusted for class of $\mathrm{VO}_{2} \%$ of predicted, and the second one for peak $\mathrm{VO}_{2}$ (absolute value), age, gender, and LVEF.

Moreover, in order to reduce the presence of confounding factors that could have affected the comparison, BMI groups were matched according to gender, age, LVEF, and peak $\mathrm{VO}_{2}$. Survival was estimated by Kaplan-Meier analysis and compared by log-rank test. Unadjusted hazard ratios and $95 \%$ confidence intervals were calculated. A $P$-value $<0.05$ was used to define statistical significance. All analyses were performed using the SAS 9.2 statistical package.

\section{Results}

Mean age of the study population was $61.6 \pm 12.6$ years, $17.2 \%$ were female, average BMI was $26.2 \pm 3.6 \mathrm{~kg} / \mathrm{m}^{2}$, LVEF $32.8 \pm 7.7 \%$, and peak $\mathrm{VO}_{2} 1129 \pm 416 \mathrm{~mL} / \mathrm{min}$. Regarding therapy, $93.7 \%$ were on ACE inhibitors or ARBs, $80.8 \%$ on beta-blockers, while $51.0 \%$ were on a mineralocorticoid antagonist. Median follow-up was $1113(553-1803)$ days; $6.8 \%$ of patients were tested with a treadmill and $93.2 \%$ with cycle-ergometer. Exercise effort was, on average, maximal or nearly maximal in all groups from a metabolic point of view, as shown by the peak exercise respiratory exchange ratio (RER; Table 1). Total and CV mortality occurred in $28.6 \%$ and $17.4 \%$, respectively, of the entire study population, including, in both cases, urgent cardiac transplant, which occurred in 110 cases.

\section{Body mass index groups}

Grouping patients according to BMI $<25$, between 25 and 30 , and between 30 and 35 showed that patients presented different clinical characteristics (Table 1a). The highest BMI group patients were younger, with more favourable LVEF, peak $\mathrm{VO}_{2}$, ventilation vs. carbon dioxide production slope, renal function, and haemoglobin level. They had a larger use of beta-blockers (Table 1a).

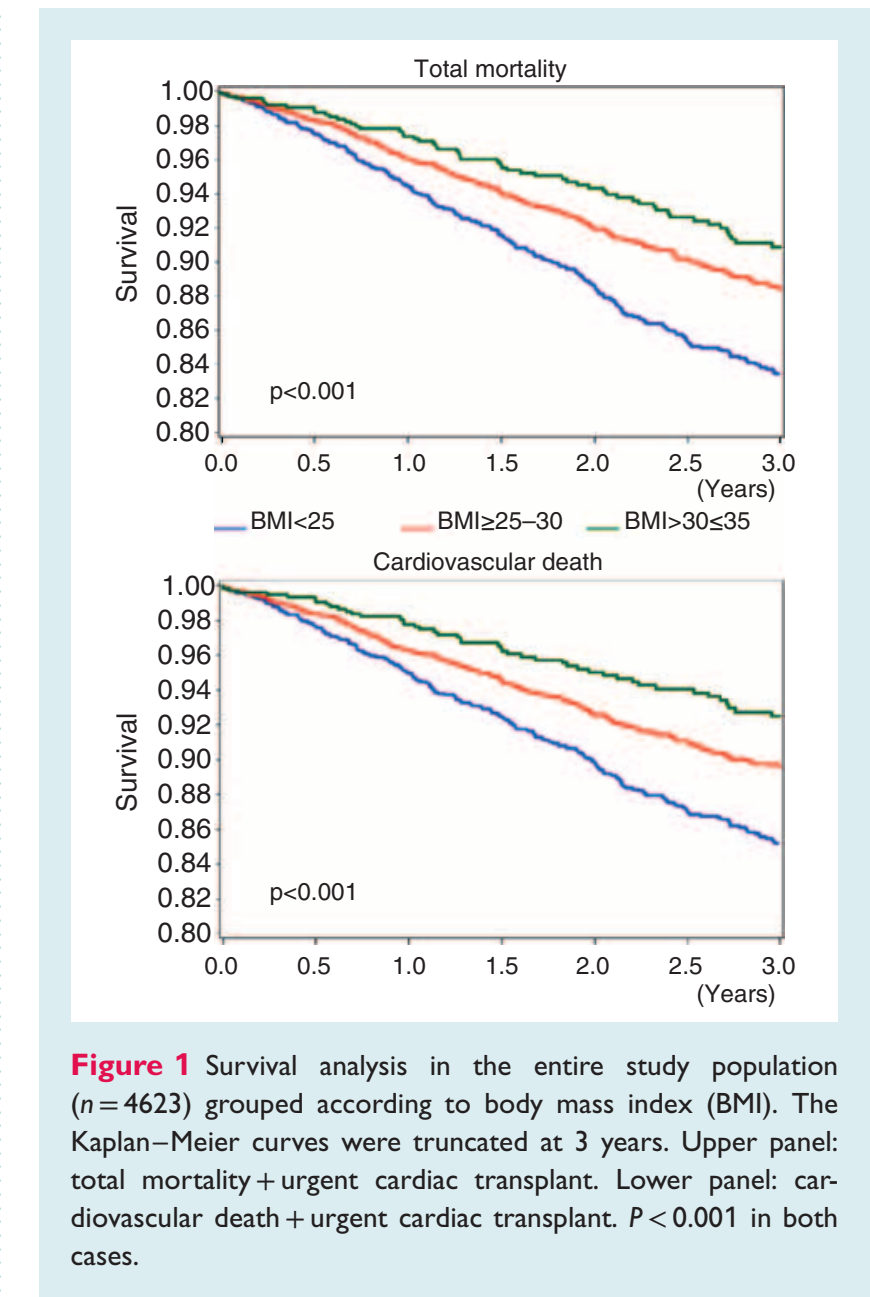

Body mass index was a significant predictor of both total and $\mathrm{CV}$ death, and the highest mortality rate occurred in the lowest BMI group $(<25)(P<0.001)$ (Figure 1 ; Table $1 b)$. Even separately analysing underweight patients $(\mathrm{BMI}<18.5)$ in the $<25 \mathrm{BMI}$ group, the results did not differ, i.e. the detrimental effects of the leaner condition were confirmed.

Interestingly, the very severely obese subjects (BMI >35), although they had more favourable clinical characteristics than the less obese (younger age, higher peak $\mathrm{VO}_{2}$, see Table 1a), presented an intermediate mortality between BMI 25-30 and BMI $>30$ to $\leq 35$ groups (Table $1 b$ ).

\section{Predicted peak oxygen consumption groups}

Peak $\mathrm{VO}_{2}$ was a significant predictor of mortality: patients with predicted peak $\mathrm{VO}_{2}<50 \%(n=1908)$ presented a worse survival than those with predicted peak $\mathrm{VO}_{2}$ ranging from $50 \%$ to $80 \%$ $(n=2363)$ and those with predicted peak $\mathrm{VO}_{2}>80 \%(n=352)$, for both total and CV deaths (Table 2).

As patients were grouped for peak $\mathrm{VO}_{2}$, BMI did not affect survival (Table 3). 


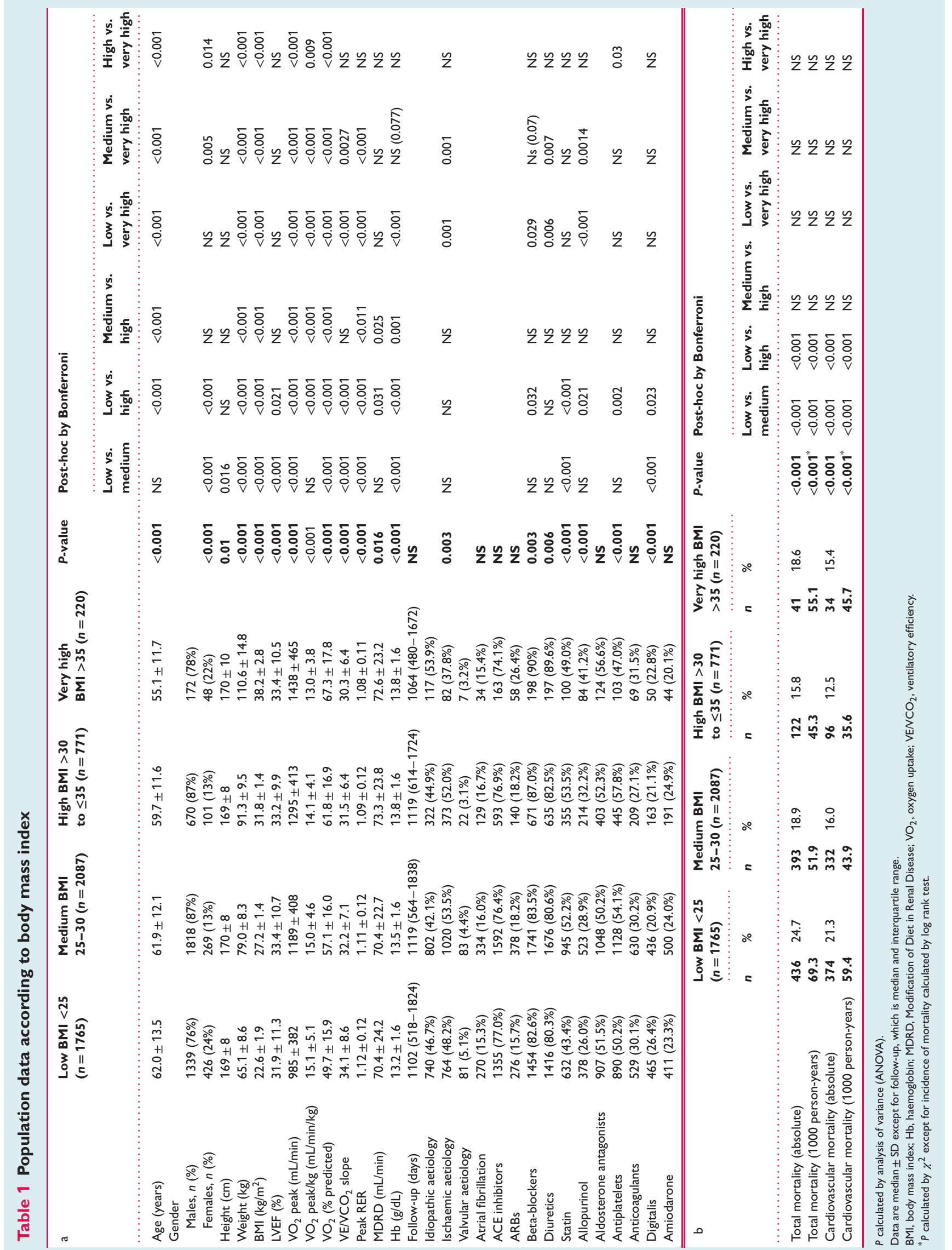


Table 2 Mortality in the heart failure population grouped according to peak oxygen consumption

\begin{tabular}{|c|c|c|c|c|c|c|c|c|c|c|}
\hline & \multicolumn{2}{|c|}{$\begin{array}{l}\mathrm{VO}_{2}<50 \% \\
(n=1908)\end{array}$} & \multicolumn{2}{|c|}{$\begin{array}{l}\mathrm{VO}_{2} 50-80 \% \\
(n=2363)\end{array}$} & \multicolumn{2}{|c|}{$\begin{array}{l}\mathrm{VO}_{2}>80 \% \\
(n=352)\end{array}$} & \multirow[t]{2}{*}{$P$-value } & \multicolumn{3}{|c|}{ Post-hoc by Bonferroni } \\
\hline & $n$ & $\%$ & $n$ & $\%$ & $\mathbf{n}$ & $\%$ & & $\begin{array}{l}\mathrm{vO}_{2}<50 \% \text { vs. } \\
\text { vo }_{2} \mathbf{5 0 - 8 0} \%\end{array}$ & $\begin{array}{l}\mathrm{VO}_{2}<50 \% v \\
\mathrm{VO}_{2}>\mathbf{8 0} \%\end{array}$ & $\begin{array}{l}\mathrm{VO}_{2} \mathbf{5 0 - 8 0} \% \\
\text { vs. } \mathrm{VO}_{\mathbf{2}}>\mathbf{8 0} \%\end{array}$ \\
\hline Total mortality (absolute) & 596 & 31.3 & 341 & 14.4 & 14 & 4.0 & $<0.001$ & $<0.001$ & $<0.001$ & $<0.001$ \\
\hline Total mortality (1000 person-years) & 89.8 & & 39.0 & & 11.8 & & $<0.001^{*}$ & $<0.001$ & $<0.001$ & $<0.001$ \\
\hline Cardiovascular mortality (absolute) & 518 & 27.2 & 274 & 11.7 & 10 & 2.8 & $<0.001$ & $<0.001$ & $<0.001$ & $<0.001$ \\
\hline Cardiovascular mortality (1000 person-years) & 78.1 & & 31.4 & & 8.5 & & $<0.001^{*}$ & $<0.001$ & $<0.001$ & $<0.001$ \\
\hline
\end{tabular}

Table 3 Mortality in the three peak oxygen consumption groups according to body mass index

\begin{tabular}{|c|c|c|c|c|c|c|c|c|}
\hline & & $n$ & $\begin{array}{l}\text { No. of events } \\
\text { total mortality }\end{array}$ & $\%$ & $\begin{array}{l}1000 \text { person- } \\
\text { years }\end{array}$ & $\begin{array}{l}\text { No. of events } \\
\text { cardiovascular } \\
\text { mortality }\end{array}$ & $\%$ & $\begin{array}{l}1000 \text { person- } \\
\text { years }\end{array}$ \\
\hline \multirow[t]{6}{*}{$\mathrm{VO}_{2}<50 \%(n=1944)$} & Low BMI $<25$ & 980 & 336 & 34.3 & 94.5 & 296 & 30.3 & 83.3 \\
\hline & Medium BMI 25-30 & 735 & 209 & $28.4^{*}$ & 86.0 & 179 & $24.4^{*}$ & 73.7 \\
\hline & High BMI $>30$ to $\leq 35$ & 193 & 51 & 26.4 & 78.4 & 43 & 22.3 & 66.1 \\
\hline & Very high BMI >35 & 36 & 16 & 44.4 & 137.2 & 13 & 36.1 & 111.5 \\
\hline & Global & & 596 & 31.3 & 89.8 & 518 & 27.2 & 78.1 \\
\hline & $P$-value & & & 0.007 & NS & & 0.008 & NS \\
\hline \multirow[t]{6}{*}{$\mathrm{VO}_{2} 50-80 \%(n=2496)$} & Low BMI $<25$ & 717 & 97 & 13.6 & 38.7 & 75 & 10.5 & 29.9 \\
\hline & Medium BMI 25-30 & 1176 & 178 & 15.2 & 39.1 & 148 & 12.6 & 32.5 \\
\hline & High $\mathrm{BMI}>30$ to $\leq 35$ & 470 & 66 & 14.0 & 39.3 & 51 & 10.9 & 30.4 \\
\hline & Very high BMI >35 & 133 & 24 & 18.0 & 51.2 & 20 & 15.2 & 42.7 \\
\hline & Global & & 341 & 14.4 & 39.0 & 274 & 11.7 & 31.4 \\
\hline & $P$-value & & & NS & NS & & NS & NS \\
\hline \multirow[t]{7}{*}{$\mathrm{VO}_{2}>80 \%(n=403)$} & Low BMI $<25$ & 68 & 3 & 4.4 & 12.9 & 3 & 4.4 & 12.9 \\
\hline & Medium BMI 25-30 & 176 & 6 & 3.4 & 10.2 & 5 & 2.8 & 8.5 \\
\hline & High $\mathrm{BMI}>30$ to $\leq 35$ & 108 & 5 & 4.6 & 13.9 & 2 & 1.9 & 5.6 \\
\hline & Very high BMI >35 & 51 & 1 & 2 & 6.3 & 1 & 2 & 6.3 \\
\hline & Global & & 14 & 4.0 & 11.8 & 10 & 2.8 & 8.5 \\
\hline & $P$-value & & & NS & NS & & NS & NS \\
\hline & Total & 4843 & 951 & 19.7 & 57.4 & 802 & 16.6 & 48.4 \\
\hline
\end{tabular}

At univariable analysis, both $\mathrm{BMI}$ and peak $\mathrm{VO}_{2}$, as absolute values and predicted values, were associated with prognosis. Cox analysis showed that BMI class adjusted for peak $\mathrm{VO}_{2} \%$ of the predicted value (class) or by age, gender, LVEF, and peak $\mathrm{VO}_{2}$ (absolute value) lost its prognostic capacity considering either total or CV death (Table 4).

When patients of the three BMl groups were matched according to age, gender, LVEF, and peak $\mathrm{VO}_{2}$ absolute value, 628 triplets of matched subjects were obtained (Table 5). No significantly different prognosis was observed for both total and CV death, regardless of the BMI group (Figure 2). It is of note that patients with BMI >35 were not included in the matching analysis due to the low sample size $(n=220)$.

\section{Discussion}

This study aimed at investigating the role of cardiorespiratory fitness in the prognosis of obese HF patients in a large cohort of stable patients undergoing CPET. Some important observations can be made. First, the obesity paradox in patients with systolic HF was also confirmed in the present group of patients referred for CPET. Secondly, the important role of cardiopulmonary exercise capacity in the prognosis of patients with systolic HF receives further confirmation. Thirdly, and most importantly, the prognostic contribution of peak $\mathrm{VO}_{2}$ overwhelms the prognostic capacity of BMI. Furthermore, the prognostic role of BMI disappears if more variables are considered, such as age, gender, LVEF, and peak $\mathrm{VO}_{2}$. Consequently, high BMI patients have a better prognosis compared 
Table 4 Mortality according to body mass index and peak oxygen consumption

\begin{tabular}{|c|c|c|c|c|}
\hline & $P$-value & Hazard ratio & $95 \% \mathrm{Cl}$ & \\
\hline \multicolumn{5}{|l|}{ CV mortality } \\
\hline BMI class unadjusted & $<0.001$ & 0.771 & $0.696-0.855$ & \\
\hline $\mathrm{VO}_{2} \%$ class unadjusted & $<0.001$ & 0.388 & $0.34-0.444$ & \\
\hline $\mathrm{VO}_{2}$ peak unadjusted $(\mathrm{mL} / \mathrm{min})$ & $<0.001$ & 0.998 & $0.998-0.999$ & \\
\hline $\mathrm{BMI}$ class adjusted by $\mathrm{VO}_{2} \%$ class & 0.3119 & 0.947 & $0.853-1.052$ & \\
\hline $\mathrm{BMI}$ class adjusted by $\mathrm{VO}_{2}$ peak, age, gender, LVEF & 0.1983 & 1.067 & $0.967-1.177$ & \\
\hline \multicolumn{5}{|l|}{ Total mortality } \\
\hline BMI class unadjusted & $<0.001$ & 0.804 & 0.732 & 0.884 \\
\hline $\mathrm{VO}_{2} \%$ class unadjusted & $<0.001$ & 0.42 & 0.372 & 0.475 \\
\hline $\mathrm{VO}_{2}$ peak unadjusted $(\mathrm{mL} / \mathrm{min})$ & $<0.001$ & 0.999 & 0.998 & 0.999 \\
\hline $\mathrm{BMI}$ class adjusted by $\mathrm{VO}_{2} \%$ class & 0.5701 & 0.973 & 0.884 & 1.07 \\
\hline $\mathrm{BMI}$ class adjusted by $\mathrm{VO}_{2}$ peak, age, gender, LVEF & 0.1983 & 1.067 & 0.967 & 1.177 \\
\hline
\end{tabular}

$\mathrm{BMI}$, body mass index; $\mathrm{CV}$, cardiovascular; $\mathrm{VO}_{2}$, oxygen consumption.

Table 5 Age, gender, left ventricular ejection fraction, and peak oxygen consumption in heart failure patients used for matching analysis

\begin{tabular}{|c|c|c|c|}
\hline & BMI $<25(n=628)$ & BMI $\geq 25-30(n=628)$ & BMI $>30$ to $\leq 35(n=628)$ \\
\hline Age (years) & $59.1 \pm 12.8$ & $59.9 \pm 11.8$ & $59.8 \pm 11.7$ \\
\hline Gender (M/F) & $(556 / 72)$ & $556 / 72$ & $556 / 72$ \\
\hline $\operatorname{LVEF}(\%)$ & $32.8 \pm 9.1$ & $32.7 \pm 9.4$ & $32.7 \pm 9.2$ \\
\hline Peak $\mathrm{VO}_{2}(\mathrm{~mL} / \mathrm{min})$ & $1227 \pm 401$ & $1240 \pm 395$ & $1242 \pm 394$ \\
\hline
\end{tabular}

BMI, body mass index; F, females; $M$, males; $\mathrm{VO}_{2}$, oxygen consumption.

with slimmer patients. However, obese groups presented a less advanced HF condition, as indicated by the presence of several more favourable prognostic indicators; they were better treated, and they presented a more preserved exercise tolerance.

Obese subjects are significantly represented in both the general and HF population. In the present cohort of HF patients able to perform a CPET, $16.3 \%$ of patients had a $\mathrm{BMI}$ ranging between 30 and 35 . Notably, we excluded from the present analysis 220 patients with extreme obesity (BMI >35), which account for $4.5 \%$ of the overall cohort of HF patients in the MECKI score database, because extreme obesity is a pathological status associated with several possible conditions, besides HF, which affect exercise capacity, including orthopaedic reasons - the work of lifting up the increased body weight, particularly on a treadmill-and mechanical difficulties in performing exercise.

However, a number of studies have now found that overweight and obese patients with HF have a better prognosis than normal weight or underweight HF patients. ${ }^{1-4}$ Also in our cohort of HF patients, high BMI patients have a better prognosis (Figure 1). Many investigators have suggested that the obesity paradox may at least partly be explained by confounding factors. ${ }^{6}$ In cohorts with CAD, patients with high levels of exercise capacity did not seem to have an obesity paradox. ${ }^{7,8}$ On the other hand, in higher risk patients with CAD and low levels of exercise tolerance, a strong obesity paradox was present, with overweight and obese individuals having a better prognosis than their lean counterparts with low exercise tolerance. $^{7-9}$

In HF, there is not only an 'obesity paradox': higher levels of blood pressure and cholesterol are also associated with a better prognosis. $^{16}$

In the present study, we evaluated a large database of HF patients with-on average-mild LV systolic dysfunction, all of which underwent an exercise test, aiming at further investigating the role of obesity in this population. We specifically investigated the role of exercise capacity vs. BMI. First of all, on average, the exercise effort performed by the patients was relevant, nearly maximal, as documented by the high RER reached at peak exercise in all BMI groups (Table 1a). ${ }^{17}$ If anything, a lower RER value was observed in the 30-35 BMI group that had the highest exercise performance. Secondly, we observed that peak $\mathrm{VO}_{2}$ maintains its prognostic value in all BMl groups, confirming the pivotal role of exercise evaluation in $\mathrm{HF}$ patients regardless of BMI. Thirdly, when peak $\mathrm{VO}_{2}$ is considered as a covariate in the survival analysis, the so-called 'obesity paradox' is lost. Regardless of whether the different BMI populations were normalized for peak $\mathrm{VO}_{2}$, either expressed as absolute value or as a percentage of the predicted value, prognostic differences between the BMI classes were lost. Indeed, we observed that BMI distinguished, in the HF population, patients with different clinical characteristics and different prognosis. Specifically, those with a higher BMI all presented better prognostic parameters, were better treated, and 

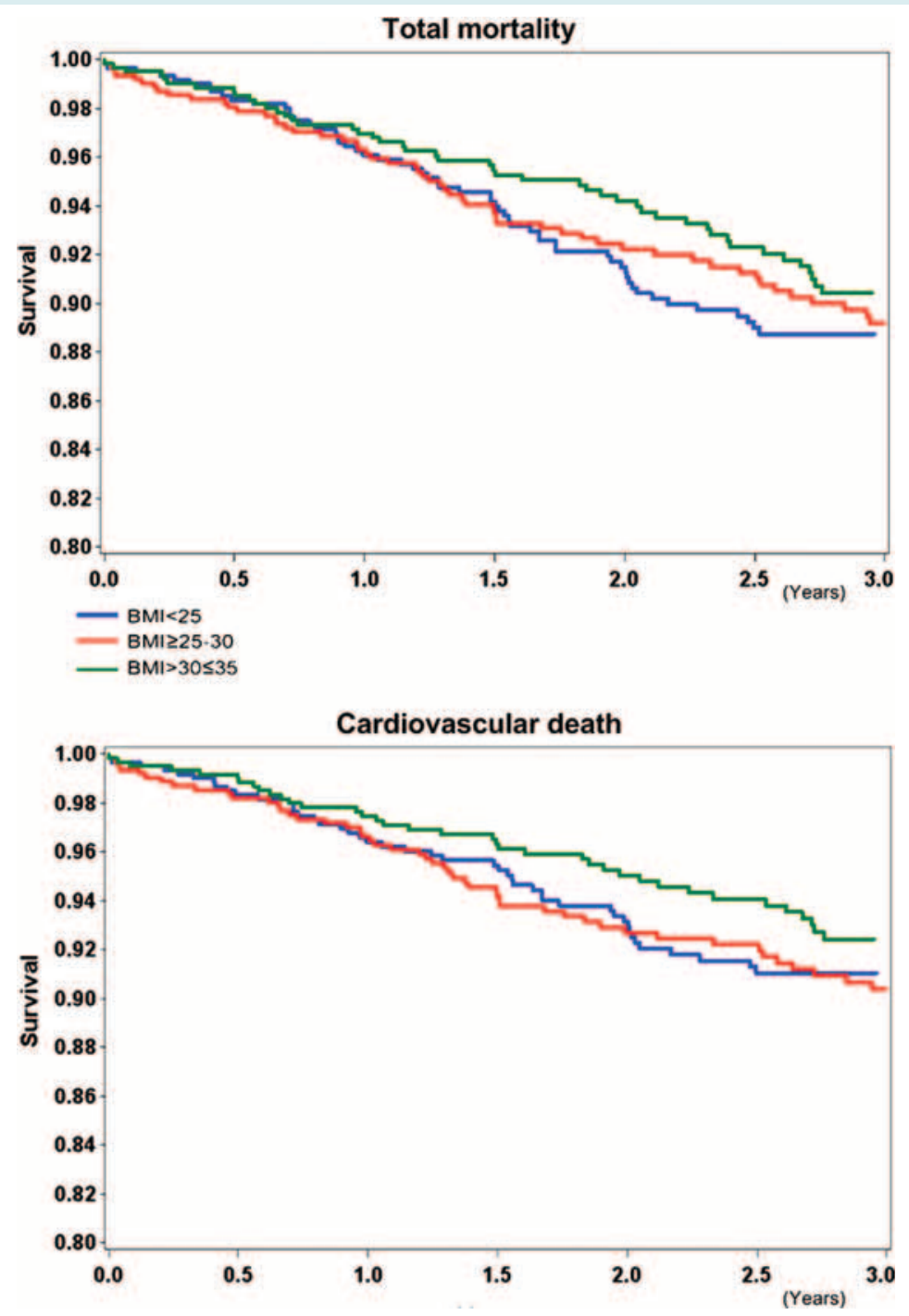

Figure 2 Survival analysis according to body mass index (BMI) in the 628 patients matched for age, gender, peak oxygen consumption, and LVEF. Upper panel: total mortality + urgent cardiac transplant. Lower panel: cardiovascular death + urgent cardiac transplant. Differences were not significant.

showed a longer survival. It is unclear why patients with high BMI and low peak $\mathrm{VO}_{2}$ were not enrolled in this database derived from the MECKI Score Research Group. It is of note that this is probably the largest HF population analysed with CPET. However, because all patients who met the MECKI score criteria were analysed, it is possible that referring physicians were reluctant to request and/or perform an exercise evaluation in obese subjects with severe HF.

To confirm the Cox analysis, we also performed a matching procedure considering, on top of peak $\mathrm{VO}_{2}$ (absolute value), age, gender, and LVEF. We were able to identify a relevant number of triplets of cases $(n=628)$ to be considered for the three BMI classes. Obese $\mathrm{HF}$ subjects matched for peak $\mathrm{VO}_{2}$, age, gender, and LVEF have a similar prognosis to that of $\mathrm{HF}$ patients with $\mathrm{BMI}<25$ or between 25 and 30 . This confirms that the 'obesity paradox' in $\mathrm{HF}$ is due to a patient selection bias.

The results presented here are consistent with recent studies performed in smaller populations of advanced systolic HF patients, showing that the obesity paradox was only observed in patients with lower cardiorespiratory fitness. ${ }^{13,18}$ Consequently, this finding supports the superior prognostic power of improved functional capacity and the importance of physical conditioning that attenuates the obesity paradox.

Progressive declines in physical activity over five decades have occurred and have primarily caused the obesity epidemic. In light of the obesity paradox, the potential value of purposeful weight loss and increased physical activity to affect levels of fitness should be underlined. ${ }^{19}$ 
Exercise training is not the single intervention that increases peak $\mathrm{VO}_{2}$ and survival, but there is evidence that cornerstone therapies such as ACE inhibitors and ARBs improve not only LV systolic function, and prognosis, but also exercise capacity (peak $\left.\mathrm{VO}_{2}\right){ }^{20,21}$ Also long-term beta-blocker therapy increases exercise time and improves ventilator response to exercise, and therefore patients are less symptomatic for a given ventilation during exercise following beta-blocker treatment. ${ }^{22,23}$

\section{Study limitations}

Some limitations of the MECKI score database have already been discussed. ${ }^{14,24}$ The patients were relatively young; mean age was 62 years, probably reflecting the inclusion of patients referred for CPET. Only $15 \%$ of the patients were females; pathophysiological and clinical gender-related differences have been found in HF. Only HF patients with reduced LVEF were included; thus, the results cannot be extrapolated to patients with preserved EF. Moreover, we evaluated patients able to perform a CPET, so that patients with very severe HF were excluded. Therefore, our findings should not be considered when evaluating patients with the above-reported characteristics. We focused on peak $\mathrm{VO}_{2}$ instead of other CPET-derived prognostic variables, because peak $\mathrm{VO}_{2}$ is the most widely used measure of exercise capacity in $\mathrm{HF}$. Further studies addressing the influence of $\mathrm{BMI}$ on $\mathrm{VE} / \mathrm{VCO}_{2}$ would be of interest. Finally, the obese subjects presented a RER without a statistically significant difference with respect to the other BMI groups $(1.08 \pm 0.12$ vs. $1.11 \pm 0.12$ high vs. medium $\mathrm{BMI})$. Accordingly exercise intensity differences, due to patients effort, are unlikely to have any clinical relevance.

\section{Conclusion}

In practical terms, these results suggest that exercise tolerance affects the relationship between BMI and survival. Thus, the obesity paradox in systolic HF is due to a patient selection bias and, at least in the present population, it does not exist. Consequently, assessing obese subjects without considering their exercise capacity, as assessed by CPET, may be misleading.

\section{Acknowledgements}

The authors are grateful to Michela Palmieri for her careful revision of the English manuscript.

Conflict of interest: none declared.

\section{Supplementary Information}

Additional Supporting Information may be found in the online version of this article:

Table $1 \mathrm{a}$ and $\mathrm{b}$. Initial study population $(\mathrm{n}=4843)$ data according to BMI.

Table 3. Mortality in the 3 peakVO2 groups according to BMI.

Figure 1. Survival according to BMI. Survival analysis in the initial entire study population $(n=4843)$ grouped according to BMI ( $<18.5$ blue line, $n=67,18.5-25$, green line, $n=1698,25-30$, black line, $n=2087$ and 30-35, grey line, $n=771,>35$, red line, $n=220$ ). The! Kaplan Meier curves were truncated at 3 years. Total mortality + urgent cardiac transplant.

\section{Appendix}

\section{Other MECKI Score Group members}

UOC Cardiologia, G da Saliceto Hospital, Piacenza: Simone Binno.

Salvatore Maugeri Foundation, IRCCS, Veruno: Pantaleo Giannuzzi, Andrea Giordano, Alessandro Mezzani.

Centro Cardiologico Monzino, IRCCS, Milano: Stefania Farina, Valentina Mantegazza, Marta Giovannardi, Anna Apostolo, Pietro Palermo, Mauro Contini, Cesare Fiorentini.

Cardiology, University of Civil Hospital, Brescia: Livio Dei Cas, Valentina Carubelli, Carlo Lombardi.

'S. Maugeri' Foundation, IRCCS, Cassano Murge: Daniela Santoro, Saba Campanale, Domenica Caputo, Andrea Passantino.

'S. Maugeri' Foundation, IRCCS, Tradate: Donatella Bertipaglia, Raffaella Vaninetti.

Ospedali Riuniti and University of Trieste: Marco Confalonieri, Elena Zambon, Emanuela Berton, Chiara Torregiani, Anna Maria lorio.

Cardiologia SUN, Ospedale Monaldi, Napoli: Teo Roselli, Andrea Buono, Raffaele Calabrò, Daniele Masarone, Giuseppe Pacileo.

Azienda Ospedaliera Sant' Andrea, 'Sapienza' Università degli Studi di Roma, Roma: Matteo Casenghi.

Cardiologia Riabilitativa, Ospedali Riuniti, Ancona: Francesca Pietrucci.

ISMETT (Mediterranean Institute for Transplantation and Advanced Specialized Therapies), Palermo: Francesco Clemenza.

UOC Cardiologia Ospedale S. Spirito, Roma: Roberto Ricci, Alessandro Ferraironi.

Federico II University, Napoli: Pasquale Perrone Filardi, Paola Gargiulo.

Division of Cardiology, Salvatore Maugeri Foundation, IRCCS, Institute of Milan, Milano: Giovanni Marchese.

S. Luca Hospital, Istituto Auxologico Italiano, Milano: Gabriella Malfatto, Sergio Caravita.

Cà Granda hospital Niguarda, Milano: Davide Girola, Fabrizio Oliva.

Salvatore Maugeri Foundation, IRCCS, Institute of Cassano Murge, Bari: Rocco La Gioia.

University of Verona, Verona: Corrado Vassanelli, Elisa Battaia.

Fondazione Gabriele Monasterio, CNR-Regione Toscana, Pisa: Luigi Pastormerlo, Michele Emdin.

\section{References}

1. Oreopoulos A, Padwal R, Kalantar-Zadeh K, Fonarow GC, Norris CM, McAlister FA. Body mass index and mortality in heart failure: a meta-analysis. Am Heart J 2008;156:13-22.

2. Horwich TB, Fonarow GC, Hamilton MA, MacLellan WR, Woo MA, Tillisch JH. The relationship between obesity and mortality in patients with heart failure. J Am Coll Cardiol 2001;38:789-795. 
3. Clark AL, Fonarow GC, Horwich TB. Waist circumference, body mass index, and survival in systolic heart failure: the obesity paradox revisited. J Card Fail 2011;17:374-380.

4. Rauchhaus M, Clark AL, Doehner W, Davos C, Bolger A, Sharma R, Coats AJ, Anker SD. The relationship between cholesterol and survival in patients with chronic heart failure. J Am Coll Cardiol 2003;42:1933-1940.

5. Kistorp C, Faber J, Galatius S, Gustafsson F, Frystyk J, Flyvbjerg A, Hildebrandt P. Plasma adiponectin, body mass index, and mortality in patients with chronic heart failure. Circulation 2005;112:1756-1762.

6. Weber MA, Neutel JM, Smith DH. Contrasting clinical properties and exercise responses in obese and lean hypertensive patients. J Am Coll Cardiol 2001;37:169-174.

7. Ades PA, Savage PD. The obesity paradox: perception vs knowledge. Mayo Clin Proc 2010;85:112-114.

8. Das SR, Alexander KP, Chen AY, Powell-Wiley TM, Diercks DB, Peterson ED, Roe MT, de Lemos JA. Impact of body weight and extreme obesity on the presentation, treatment, and in-hospital outcomes of 50,149 patients with ST-segment elevation myocardial infarction results from the NCDR (National Cardiovascular Data Registry). J Am Coll Cardiol 2011;58: 2642-2650.

9. Kodama S, Saito K, Tanaka S, Maki M, Yachi Y, Asumi M, Sugawara A, Totsuka $\mathrm{K}$, Shimano H, Ohashi Y, Yamada N, Sone H. Cardiorespiratory fitness as a quantitative predictor of all-cause mortality and cardiovascular events in healthy men and women: a meta-analysis. JAMA. 2009;301:2024-2035.

10. Mancini DM, Eisen H, Kussmaul W, Mull R, Edmunds LH Jr, Wilson JR. Value of peak exercise oxygen consumption for optimal timing of cardiac transplantation in ambulatory patients with heart failure. Circulation 1991;83:778-786.

11. Romero-Corral A, Montori VM, Somers VK, Korinek J, Thomas RJ, Allison TG, Mookadam F, Lopez-Jimenez F. Association of bodyweight with total mortality and with cardiovascular events in coronary artery disease: a systematic review of cohort studies. Lancet 2006;368:666-678.

12. Goel K, Thomas RJ, Squires RW, Coutinho T, Trejo-Gutierrez JF, Somers VK, Miles JM, Lopez-Jimenez F. Combined effect of cardiorespiratory fitness and adiposity on mortality in patients with coronary artery disease. Am Heart J 2011;161:590-597.

13. Lavie CJ, Cahalin LP, Chase P, Myers J, Bensimhon D, Peberdy MA, Ashley E, West E, Forman DE, Guazzi M, Arena R. Impact of cardiorespiratory fitness on the obesity paradox in patients with heart failure. Mayo Clin Proc 2013;88: $251-258$.

14. Agostoni P, Corra U, Cattadori G, Veglia F, La Gioia R, Scardovi AB, Emdin M, Metra M, Sinagra G, Limongelli G, Raimondo R, Re F, Guazzi M, Belardinelli R, Parati G, Magri D, Fiorentini C, Mezzani A, Salvioni E, Scrutinio D, Ricci R, Bettari
L, Di Lenarda A, Pastormerlo LE, Pacileo G, Vaninetti R, Apostolo A, lorio A, Paolillo S, Palermo P, Contini M, Confalonieri M, Giannuzzi P, Passantino A, Cas LD, Piepoli MF, Passino C. Metabolic exercise test data combined with cardiac and kidney indexes, the MECKI score: a multiparametric approach to heart failure prognosis. Int J Cardiol 2013;167:2710-2718.

15. Hansen JE, Sue DY, Wasserman K. Predicted values for clinical exercise testing. Am Rev Respir Dis 1984;129:S49-S55.

16. Lavie CJ, Mehra MR, Milani RV. Obesity and heart failure prognosis: paradox or reverse epidemiology? Eur Heart J 2005;26:5-7

17. Corra U, Piepoli MF, Adamopoulos S, Agostoni P, Coats AJ, Conraads V, Lambrinou E, Pieske B, Piotrowicz E, Schmid JP, Seferovic PM, Anker SD, Filippatos G, Ponikowski PP. Cardiopulmonary exercise testing in systolic heart failure in 2014: the evolving prognostic role: a position paper from the Committee on Exercise Physiology and Training of the Heart Failure Association of the ESC. Eur J Heart Fail 2014;16:929-941.

18. Clark AL, Fonarow GC, Horwich TB. Impact of cardiorespiratory fitness on the obesity paradox in patients with systolic heart failure. Am J Cardiol 2015;115:209-213.

19. Lavie CJ, McAuley PA, Church TS, Milani RV, Blair SN. Obesity and cardiovascular diseases: implications regarding fitness, fatness, and severity in the obesity paradox. J Am Coll Cardiol 2014;63:1345-1354.

20. Guazzi M, Palermo P, Pontone G, Susini F, Agostoni P. Synergistic efficacy of enalapril and losartan on exercise performance and oxygen consumption at peak exercise in congestive heart failure. Am J Cardiol 1999;84:1038-1043.

21. de Vries RJ, Quere M, Lok DJ, Sijbring P, Bucx JJ, van Veldhuisen DJ, Dunselman $\mathrm{PH}$. Comparison of effects on peak oxygen consumption, quality of life, and neurohormones of felodipine and enalapril in patients with congestive heart failure. Am J Cardiol 1995; 76:1253-1258.

22. Witte KK, Thackray S, Nikitin NP, Cleland JG, Clark AL. The effects of long-term beta-blockade on the ventilatory responses to exercise in chronic heart failure. Eur J Heart Fail 2005;7:612-617.

23. Kataoka M, Satoh T, Yoshikawa T, Nakamura I, Kohno T, Yoshizawa A, Anzai $\mathrm{T}$, Ogawa S. Comparison of the effects of carvedilol and metoprolol on exercise ventilatory efficiency in patients with congestive heart failure. Circ J 2008;72:358-363.

24. Agostoni P, Corra U, Cattadori G, Veglia F, Battaia E, La Gioia R, Scardovi $A B$, Emdin M, Metra M, Sinagra G, Limongelli G, Raimondo R, Re F, Guazzi M, Belardinelli R, Parati G, Magri D, Fiorentini C, Cicoira M, Salvioni E, Giovannardi M, Mezzani A, Scrutinio D, Di Lenarda A, Mantegazza V, Ricci R, Apostolo A, lorio A, Paolillo S, Palermo P, Contini M, Vassanelli C, Passino C, Piepoli MF. Prognostic value of indeterminable anaerobic threshold in heart failure. Circ Heart Fail 2013;6:977-987. 